\title{
El embarazo humano: paradigma biológico de tolerancia y adaptación
}

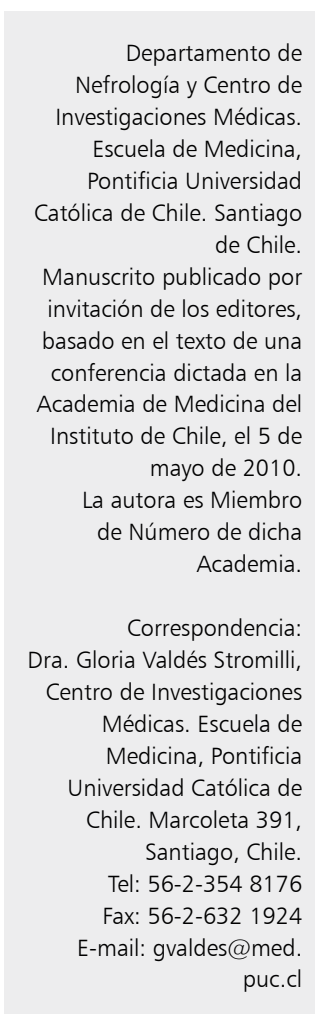

GLORIA VALDÉS S.

\section{Human pregnancy, a biological paradigm of tolerance and adaptation}

This review analyses the changes in immunological tolerance, and the systemic and local hemodynamic changes observed along human pregnancy. To underscore the conceptual importance of tolerance and adaptation the background is provided by the two main advocates of these ideas: Gandhi and Darwin. The cognate factors that determine immunological tolerance (IT), systemic (SA) and local adaptation (LA) are multiple; IT = desensitisation to paternal antigens, absence of HLA-A, roles of HLA-G, natural killer cells and their receptors; $S A=$ decreased vascular resistance, plasma volume expansion, increased cardiac output and plasma renin activity; $L A=$ prostacyclin, nitric oxide, kallikrein-kinin system, vasodilator arm of the renin-angiotensin system, vascular endothelial growth factor (VEGF). A possible role of vasodilators in the crucial process of trophoblast invasion and uterine artery transformation is supported. The relevance of an adequate adaptation to pregnancy is highlighted not only by the intragestational complications derived from a defective process, such as intrauterine growth restriction, preterm birth, and preeclampsia-its foremost expression- but also by the long term cardiovascular complications of the mother and her offspring.

(Rev Med Chile 2011; 139: 400-405).

Key words: Adaptation, physiologic; Immune tolerance; Maternal-fetal exchange; Pregnancy.
$\mathrm{E}$ ste trabajo analiza la importancia primordial de la tolerancia y la adaptación en los procesos biológicos que se desarrollan a lo largo del embarazo humano. Para enfatizar la fuerza y el significado de los conceptos de tolerancia y adaptación quiero hacerlo con el trasfondo de su sentido más amplio, empleando los ejemplos de sus dos máximos exponentes, Mahatma Gandhi y Charles Darwin (Figura 1).

Tolerancia deriva del griego talao (tolerar, sufrir, aguantar). En el siglo XIV se le dio el significado de "permisivo", en el XVIII de "libre de fanatismo o severidad", en el XIX como "el rango de variación permitida", y para la fisiología como "la habilidad de aceptar dosis altas". Si bien el primero en emplear políticamente el concepto de tolerancia-no violencia fue Gandhi, es imperioso considerar que éste concepto nació de las enseñanzas fundamentales de Jesucristo, de amar al prójimo como a uno mismo y de poner la otra mejilla, que en palabras muy simples hacen desaparecer la diferencia y la confrontación entre el yo y el otro, o entre el self y el non-self que actualmente se utilizan en las áreas de la psicología y la inmunología. Mahatma Gandhi (1869-1948), hindú graduado de abogado en Inglaterra introdujo, el concepto de "tolerancia-no violencia" para combatir los severos problemas de casta, religiosos y políticos, que afectaban a la India en la primera mitad del siglo XX (la discriminación de los intocables, la lucha entre hindúes y musulmanes, la dominación inglesa). Para él la no-violencia era incomprensible si no se interpretaba como fruto de unidad interna y como forma de vida, y no solamente 

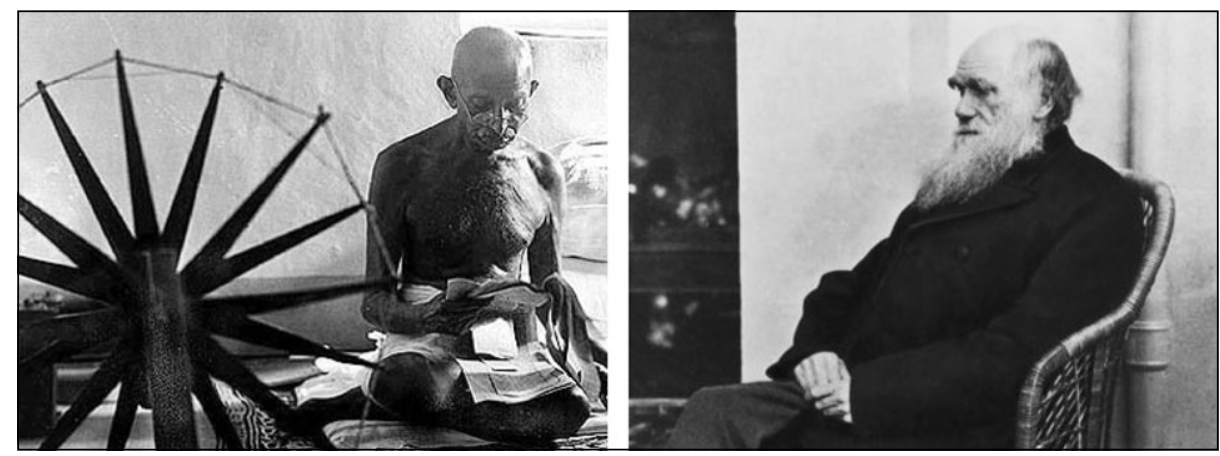

Figura 1. Mohandas Gandhi y Charles Darwin, los más representativos preconizadores de la tolerancia y la adaptación.

como una táctica política eficaz para liberar a la India del dominio extranjero, y para concentrarse posteriormente a desarrollar su identidad nacional. El juramento que desde 1919 hacía tomar a sus seguidores representa sus ideas de igualdad, hermandad, solidaridad y respeto entre los hombres, y la necesidad de borrar las diferencias y el sectarismo entre yo y los otros:

"Con Dios como testigo, Hindúes, Musulmanes, Siks, Parsis y Cristianos declaramos que debemos tratarnos como hermanos, que no habrá diferencias entre nosotros, que las tristezas de uno serán las del otro, y que cada uno ayudará a aliviarlas. Respetaremos la religión del otro y sus sentimientos, y no impediremos sus prácticas. Siempre nos abstendremos de la violencia entre nosotros por motivos religiosos."

La validez de la tolerancia política se confirmó en 1947 con la independencia de la India del inmenso poder que en ese tiempo tenía el Imperio Británico. Posteriormente se demostró su vigencia con el logro de Martín Luther King de integrar a los afroamericanos con plenos derechos a la sociedad de los Estados Unidos, y con la desaparición del apartheid, lograda en 1990 por Nelson Mandela, ícono actual de la no violencia. Ojalá que la resistencia no violenta de Aung San Suu Kyi y de Lui Xiabo en Birmania y China contribuya a derrotar los regímenes dictatoriales de ambos países.

Adaptación deriva del latín ad (hacia) y aptus (adecuado, ajustado). A fines del siglo XVIII se le dio el sentido de "modificación para ajustarse a una nueva condición", y su sentido biológico fue empleado por primera vez por Darwin, en 1859. Charles Darwin, clérigo con dos años de estudios médicos en la Universidad de Edimburgo y naturalista de vocación, formó parte de la segunda expedición del bergantín Beagle, entre 1831 y $1836^{2}$. Vale la pena destacar que en este período tenía 22 a 27 años, por lo que sorprenden sus agudas y acuciosas observaciones sobre geología, geografía, aspecto y conductas de los hombres primitivos, flora, fauna, y vida política de cada país, entre otros. En las diferentes islas Galápagos observa la gradualidad y diversidad de los cambios del pico en diucas y especies afines. En 1845, a los 37 años, postuló que estas diferencias estructurales en un grupo de aves pequeño e íntimamente relacionado se deben a que una especie fue tomada y modificada, con diversos fines. Este pensamiento inicial fue adquiriendo fuerza en los años posteriores y terminó generando su teoría sobre el origen de las especies a través de selección natural, cuya publicación -en 1859- revolucionó no solamente a la biología sino a la creencia que cada especie había sido creada individualmente. Con su formación de clérigo, nadie mejor que Darwin entendía la repercusión de su teoría sobre las creencias religiosas, y la difusión de esta teoría fue diferida, por temor tanto a la reacción de la sociedad inglesa como a las repercusiones en su propio grupo familiar. Es difícil imaginar la reacción a este "Big Bang" conceptual para los que crecimos creyendo en la adaptación biológica, que en la actualidad ha sido sustentada por la caracterización preliminar del genoma del hombre de Neandertal ${ }^{3}$.

Si bien la tolerancia tiene un sentido preferentemente espiritual, y la adaptación uno más estructural, ambas comparten características comunes. Entre éstas, simpleza, percepción o in- 
tuición, grandeza, valentía, plasticidad para aceptar al otro como igual, y para modificar nuestro pensamiento y estructura. Otro importante factor común entre ellas es la destrucción que genera la falta de tolerancia o de adaptación, entre las que se pueden contar las violentas manifestaciones en Soweto, el asesinato de Gandhi, la desaparición de los dinosaurios y, más recientemente, los efectos del terremoto del 27 de febrero de 2010 en Chile. A la intolerancia se agrega la capacidad de entrar en un círculo vicioso, que genera una destrucción creciente que se hace imposible manejar.

Igual que la falta de adaptación de las especies y de tolerancia en las sociedades llevan a destrucción, la falla en la tolerancia y adaptación al embarazo causa abortos, mortinatos, desprendimiento placentario, desnutrición intrauterina, parto prematuro y preeclampsia (hipertensión más proteinuria) y sus formas más severas (eclampsia y síndrome de HELLP). En la preeclampsia, la forma más frecuente de las patologías reproductivas, se han observado fallas de mecanismos individuales, lo que implica que esta importante complicación del embarazo representa un síndrome y no una enfermedad. La falla primaria -el déficit de la transformación de las arterias espirales por el trofoblasto- ocurre antes de las 20 semanas de gestación y no tiene expresión clínica. La placenta isquémica posteriormente deporta a la circulación materna micropartículas de sinciciotrofoblasto y factores que provocan una disfunción endotelial sistémica que genera la hipertensión, el edema, la proteinuria, el consumo plaquetario, el compromiso de la circulación cerebral ${ }^{4}$. Así, el síndrome bifásico de la preeclampsia representa un ejemplo del círculo vicioso causado por la falta de tolerancia y de adaptación, contra el cual el manejo clínico habitual con antihipertensivos solo ayuda a reducir la mortalidad materna derivada de la hipertensión. Christopher Redman, experto investigador inglés en esta área, afirmó:

\footnotetext{
"En términos evolutivos, la preeclampsia puede ser percibida como una lucha entre las necesidades de sobrevida de los genes maternos y paternos (fetales) o como un conflicto materno fetal. Se sugiere que la hipertensión de la preeclampsia está determinada por el feto (placenta) para ganar una mayor fracción de la circulación materna. El precio en término de riesgo de mortalidad materna parece ser aceptable desde el punto de vista evolutivo"4.
}

\section{Embarazo y tolerancia inmunológica}

Antes de analizar la tolerancia biológica de la madre al feto, es importante recordar que si un hijo dona un riñón a su madre, el órgano tiene mayores posibilidades de sufrir un rechazo hiperagudo o agudo y tiene una menor sobrevida a 1 y 5 años, a pesar del tratamiento inmunosupresor ${ }^{5}$. ¿Qué permitió que este mismo hijo viviera en el vientre materno sin ser rechazado cuando la placenta fetal estaba directamente en contacto con la decidua materna a lo largo de todo el embarazo?

En primer lugar, se ha comprobado un mayor riesgo de preeclampsia para el embarazo que resulta de una sola relación sexual con el padre, o de una convivencia breve $e^{6}$, lo que probablemente es secundario a una falta de desensibilización de la madre a los antígenos paternos. Me pregunto si la asociación de una mejor tolerancia biológica al aumentar el tiempo de convivencia de sus padres representa un mecanismo adaptativo para mejorar la sobrevida de la especie, ya que esto contribuiría a una unión más consolidada de los padres biológicos.

Además, desde el punto de vista fetal, el trofoblasto no expresa el principal antígeno de histocompatibilidad: el antígeno leucocitario humano (HLA)-A. En cambio, expresa HLA-G, especialmente HLA-G5, que tiene por función atenuar la respuesta inmunológica materna ${ }^{7}$.

También contribuye a crear una zona de privilegio, tregua, silencio o tolerancia inmunológica para el feto la acción de las células natural killer (NK) de la decidua, distintas a las de sangre periférica. A diferencia de las NK circulantes, que capturan células tumorales o infectadas, las NK deciduales activadas producen citoquinas que protegen al trofoblasto y favorecen su invasión al ser activadas (Figura 2); presentan receptores para inmunoglobulinas (killer immunoglobulin-like receptors o KIR), que reconocen en el trofoblasto al HLA-C paterno. Los haplotipos de KIR son A y B, de los cuales el B tiene receptores activantes adicionales. El genotipo materno puede ser AA (no activante), $\mathrm{AB}$ o BB (ambos con uno a cinco KIRs activantes). Los ligandos para KIR en el trofoblasto pueden ser $\mathrm{C} 1$ y C2. Las combinaciones de KIR maternos y HLA-C fetales pueden ser favorables o desfavorables a la invasión del trofoblasto, sean (AA con $\mathrm{C} 1+\mathrm{C} 1, \mathrm{AB}$ y $\mathrm{BB}$ con las 3 combinaciones de $\mathrm{C}$ ) o (AA con $\mathrm{C} 1+\mathrm{C} 2$ o $\mathrm{AA}$ con $\mathrm{C} 2+\mathrm{C} 2$ ), respectivamente $^{8}$ 


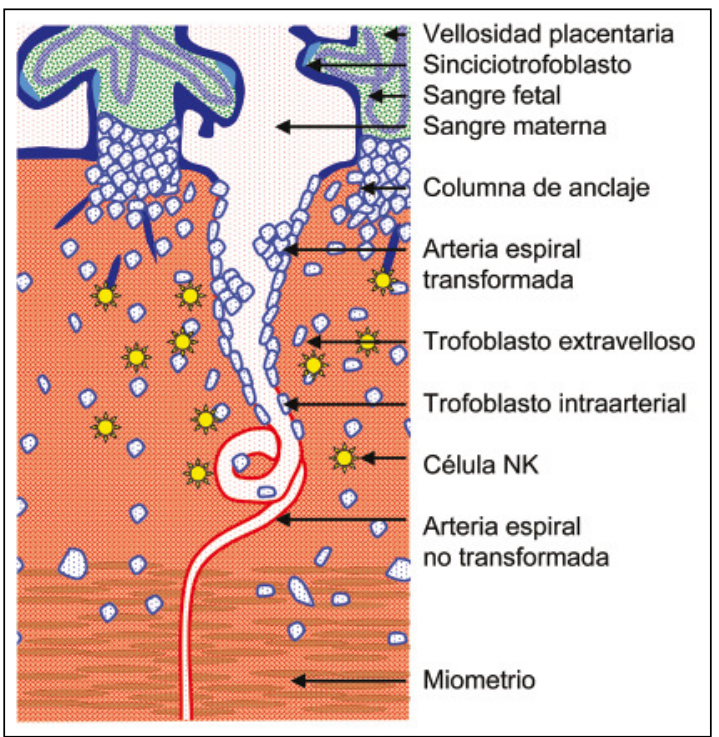

Figura 2. Esquema de la interfase materno-fetal, que destaca la cercanía de la sangre materna con la fetal, y el trayecto del trofoblasto desde la columna de anclaje a la arteria espiral. Las células estrelladas son las natural killers (NK), que contribuyen a neutralizar la diferencia inmunológica del trofoblasto y facilitan su invasión.

Vemos así como cambios en la reacción inmunológica al embarazo anulan las diferencias entre trofoblasto y células maternas, y se abstienen de la violencia entre estos tipos celulares, como lo planteó Gandhi para los distintos grupos religiosos de hindúes.

\section{Embarazo y adaptaciones hemodinámicas sistémicas y locales}

Analizaré a continuación las adaptaciones hemodinámicas sistémicas de la madre:

A lo largo del embarazo, la madre aumenta su débito cardíaco en prácticamente $1 \mathrm{~L} / \mathrm{min} / \mathrm{m}^{2}$, el volumen plasmático en $600 \mathrm{ml}$, y la actividad de renina plasmática en $10 \mathrm{ng} / \mathrm{ml} / \mathrm{h}$. Estos cambios que, aislados, pueden en una mujer no embarazada provocar elevación de las cifras tensionales (eg. hipertiroidismo, insuficiencia renal, estenosis renovascular), se acompañan en la gestación incluso de una reducción de la presión arterial en el segundo trimestre. Sin duda que esta adaptación se debe a un incremento de los sistemas vasodilatadores, como el calicreína-cininas, la vía de L-arginina-óxido nítrico, las prostaglandinas vasodilatadoras, la vía vasodilatadora del sistema renina-angiotensina (angiotensina-(1-7) y la enzima ACE2 que la genera a partir de angiotensina I $\mathrm{y}$ al factor de crecimiento vascular (vascular endotelial growth factor o VEGF) y sus receptores ${ }^{9}$ (Figura 3). Interesantemente, en embarazadas normales hemos podido comprobar que mientras la calicreína urinaria alcanza sus máximos valores entre las 8 y las 12 semanas de amenorrea, para caer después, angiotensina-(1-7) se eleva progresivamente, sugiriendo un mecanismo de relevo entre los vasodilatadores, fundamental para la mantención de vasodilatación bajo los perfiles hormonales cambiantes del embarazo. La observación de una reducción de la resistencia de la arteria pulmonar en una paciente nuestra con hipertensión primaria, confirma la potencia vasodilatadora del embarazo'.

Aparte de la vasodilatación sistémica materna, es necesario que una elevada proporción del

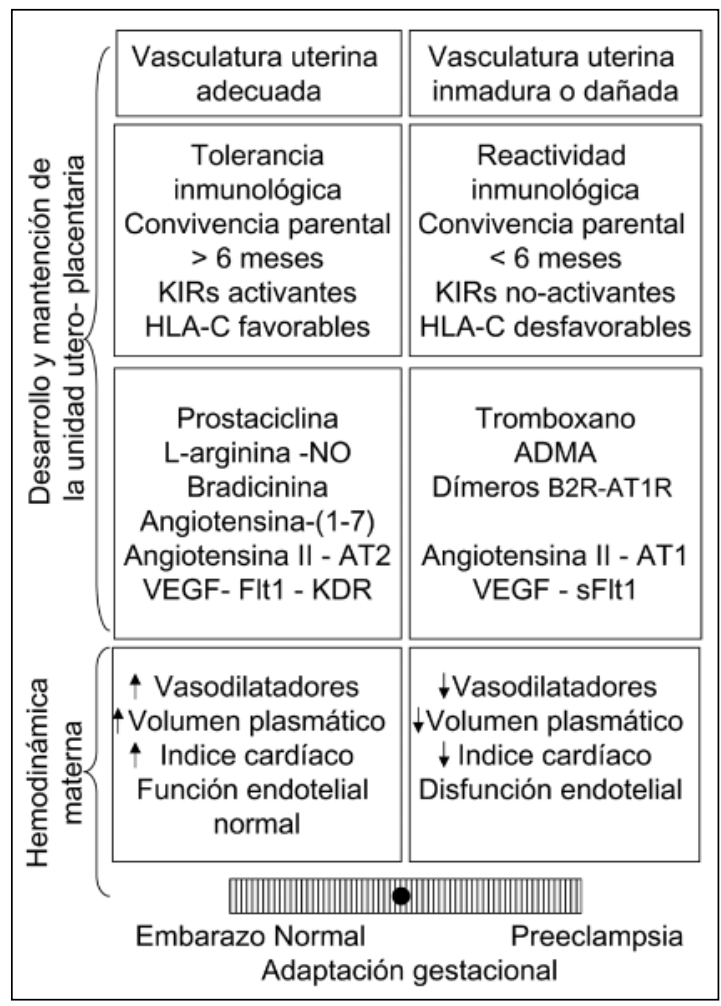

Figura 3. Balance de factores que intervienen en la tolerancia y adaptación del embarazo, tanto desde el punto de vista sistémico como local, y que determinan una gestación normal o preeclámptica. 
débito cardíaco de la madre y su hijo establezcan una proximidad permanente, que permita el intercambio de oxígeno, nutrientes y desechos. La transformación de las arterias espirales del útero materno en vasos que pierden su conformación tubular y su capa muscular, para convertirse en vasos saculares de amplio lumen sin reactividad a estímulos vasoactivos, representa la mayor adaptación morfológica materna (Figura 2) ${ }^{10,11}$. Postulamos que los vasodilatadores del trofoblasto tienen un rol en la preparación de las arterias espirales y, posteriormente, en la mantención del flujo inter e intravelloso y en la prevención de trombosis de la superficie del sinciciotrofoblasto. Esta hipótesis se apoya en la expresión de calicreína, receptor de bradicinina, angiotensina-(1-7) ACE2, sintasa de NO, ciclooxigenasa, VEGF y su receptor KDR, en el trofoblasto intraarterial, el sinciciotrofoblasto, y el endotelio de los capilares fetales intravellosos?. Si bien no hemos podido encontrar una diferencia en la expresión de factores vasodilatadores en la preclampsia, debemos considerar que las muestras de tejidos reproductivos fueron tomadas alejadas de la etapa de invasión trofoblástica, cuyo defecto determina el desarrollo posterior del daño multisistémico de la preeclampsia.

La co-localización de los factores vasodilatadores, su interrelación funcional, y la conversión del efecto vasoconstrictor de la estimulación del receptor 1 de angiotensina II (AT1) dan énfasis a la importancia de la adaptación vasodilatadora.

Como podemos apreciar, además de la creación de la placenta, tanto a nivel del aparato cardiovascular de la madre y del útero materno se lleva a cabo una marcada adaptación multifactorial para asegurar la mejor perfusión del feto (Figura 3). La magnitud de estas adaptaciones constituye "un gran y rápido salto" adaptativo que sobrepasa en magnitud y velocidad a las "leves variaciones sucesivas" mediante las que Darwin explica la selección natural ${ }^{12}$.

\section{Implicancias a largo plazo de una adaptación adecuada o inadecuada al embarazo}

Es importante considerar que la falta de adaptación no sólo se expresa durante el embarazo, sino que posteriormente marca a la madre y al hijo.

Desde el punto de vista materno existen evidencias que asocian mayor riesgo cardiovascular a la presentación de preeclampsia, a otras formas de hipertensión en el embarazo y a patologías asociadas a defectos de la placentación. Por esto se considera al embarazo como una "prueba de esfuerzo" para la salud cardiovascular de la ma$\mathrm{dre}^{13,14}$. En un estudio realizado por nuestro grupo, en mujeres sometidas a angiografía coronaria por sospecha clínica de enfermedad coronaria, encontramos que aquellas con antecedente de hipertensión en el embarazo presentaban un mayor aumento del número de arterias estenosadas a medida que avanza la edad, que en las pacientes sin hipertensión en el embarazo ${ }^{9}$. Ya que los riesgos CV aumentan a lo largo de un continuo, es muy probable que la asociación de embarazo hipertensivo y enfermedad CV posterior esté determinado por factores de riesgo en rango normal alto en la etapa reproductiva, que posteriormente se ven agravados por persistencia o incremento de la influencia ambiental. Por esta razón es fundamental incorporar la historia de los embarazos como un índice predictor que permite una intervención precoz.

Para el hijo, desarrollarse en un ambiente favorable o desfavorable también influye sobre su salud. Barker desarrolló, en 1992, la teoría denominada fetal programming (actualmente denominada developmental origins of adult disease) para describir las observaciones epidemiológicas que asocian en forma inversa un bajo peso de nacimiento con enfermedades del adulto, como enfermedad coronaria, hipertensión arterial, diabetes ${ }^{15}$. Esta asociación se explicaría por adaptaciones del feto a un ambiente desfavorable, cambiando las concentraciones de hormonas fetales y placentarias, $y$ adquiriendo un fenotipo "ahorrador" (thrifty). Se ha demostrado que las enfermedades del adulto se ven acentuadas en los nacidos de bajo peso que de un ambiente desfavorable pasan a uno de abundancia compensatoria. Finalmente, se postula que la asociación entre vida intra y extrauterina puede estar determinada por cambios epigenéticos, o modificación de función de los genes no acompañadas de cambios en su secuencia pero transmitidos de una generación a otra, determinados por la relación entre genes y ambiente.

\section{Conclusión}

El maravilloso proceso del embarazo humano normal representa un paradigma de tolerancia y adaptación, que concuerda con las derivaciones 
etimológicas y las definiciones sociales y evolutivas de estos conceptos. El enfoque más amplio de los cambios provocados por el embarazo me hace tener la profunda convicción que si el producto de un embarazo normal ha sido sujeto a una cuidadosa selección natural, eliminarlo in utero por falta de tolerancia y adaptación a la llegada de un nuevo hijo perturba biológica y espiritualmente la evolución de la humanidad.

Agradecimientos: Los estudios de la autora y su grupo, sobre factores vasodilatadores en el embarazo humano, han sido apoyados por proyectos financiados por el Fondo Nacional de Ciencia y Tecnología (FONDECYT 1020705, 1050707, 1080228).

\section{Referencias}

1. Radhakrishnan N. Gandhi: Quest for tolerance and survival. New Delhi: Gandhi Media Centre; 1995.

2. Darwin C. The voyage of the Beagle: Journal of researches into the natural history and geology of the countries visited during the voyage of H.M.S. Beagle round the world. Modern Library Publishers; 1945.

3. Green RE, Krause J, Briggs AW, Maricic T, Stenzel U, Kircher M, et al. A draft sequence of the Neandertal genome. Science 2010; 328: 710-22.

4. Redman CW, Sargent IL. Latest advances in understanding preeclampsia. Science 2005; 308: 1592-4.

5. Ghafari A. Offspring-to-mother and husband-to-wife renal transplantation: A single-center experience. Transplant Proc 2008; 40: 140-2.

6. Kho EM, McCowan LM, North RA, Roberts CT, Chan E, Black MA, et al. Duration of sexual relationship and its effect on preeclampsia and small for gestational age perinatal outcome. J Reprod Immunol. 2009;82:66-73

7. Hunt JS. Stranger in a strange land. Immunol Rev 2006; 213: 36-47.

8. Moffett A, Hiby SE. How does the maternal immune system contribute to the development of pre-eclampsia? Placenta 2007; 28 Suppl A: S51-56.

9. Valdés G, Matthei R, Fernández MS, Schacht C, Corthorn J, Germain AM. Hipertensión pulmonar y embarazo. Rev Med Chile 2002; 130: 201-8.

10. Kaufmann P, Black S, Huppertz B. Endovascular trophoblast invasion: Implications for the pathogenesis of intrauterine growth retardation and preeclampsia. Biol Reprod 2003; 69: 1-7.

11. Red-Horse K, Zhou Y, Genbacev O, Prakobphol A, Foulk R, McMaster M, et al. Trophoblast differentiation during embryo implantation and formation of the maternalfetal interface. J Clin Invest 2004; 114: 744-54.

12. Darwin C. On the origin of species by means of natural selection, or the preservation of favoured races in the struggle for life London: John Murray; 1859.

13. Williams D. Pregnancy: A stress test for life. Curr Opin Obstet Gynecol 2003;15: 465-71.

14. Irgens HU, Reisaeter L, Irgens LM, Lie RT. Long term mortality of mothers and fathers after pre-eclampsia: Population based cohort study. BMJ 2001; 323: 1213-7.

15. Barker DJ. Fetal growth and adult disease. Br J Obstet Gynaecol 1992; 99: 275-6. 